\title{
Image guided adaptive brachytherapy for cervical cancer: dose contribution to involved pelvic nodes in two cancer centers
}

\author{
Willemien van den Bos, MD', Sushil Beriwal, MD², Laura Velema, MD³, Astrid A.C. de Leeuw, PhD', Christel N. Nomden, \\ BHS', Ina-M. Jürgenliemk-Schulz, MD, PhD' \\ 'Department of Radiation Oncology, University Medical Center Utrecht, Utrecht, The Netherlands, ${ }^{2}$ Department of Radiation Oncology. \\ University of Pittsburgh Cancer Institute, Pittsburgh, PA, USA, ${ }^{3}$ Department of Radiation Oncology. Erasmus Medical Center, Rotterdam, \\ The Netherlands
}

\begin{abstract}
Purpose: The goal of this study was to determine the dose contributions from image guided adaptive brachytherapy (IGABT) to individual suspicious pelvic lymph nodes (pLNN) in cervical cancer patients. Data were collected in two cancer centers, University of Pittsburgh Cancer Institute (UPCI) and University Medical Center Utrecht (UMCU).

Material and methods: 27 and 15 patients with node positive cervical cancer treated with HDR (high dose rate) or PDR (pulsed dose rate)-IGABT were analyzed. HDR-IGABT (UPCI) was delivered with CT/MRI compatible tandem-ring applicators with 5.0-6.0 Gy $\times$ five fractions. PDR-IGABT (UMCU) dose was delivered with Utrecht tandem-ovoid applicators with $32 \times 0.6 \mathrm{~Gy} \times$ two fractions. Pelvic lymph nodes with short axis diameter of $\geq 5 \mathrm{~mm}$ on pre-treatment MRI or PET-CT were contoured for all BT-plans. Dose contributions to individual pLNN expressed as $\mathrm{D}_{90}$ (dose to $90 \%$ of the volume) were calculated from dose-volume histograms as absolute and relative physical dose ( $\%$ of the reference dose) for each fraction. For each node, the total dose from all fractions was calculated, expressed in EQD2 (equivalent total dose in 2 Gy fractions).

Results: Fifty-seven (UPCI) and 40 (UMCU) individual pLNN were contoured. The mean $\mathrm{D}_{90} \mathrm{pLNN}$ was $10.8 \%$ (range 5.7-25.1\%) and 20.5\% (range 6.8-93.3\%), respectively, and therefore different in the two centers. These values translate into 2.7 Gy (1.3-6.6 Gy) EQD2 and 7.1 Gy (2.2-36.7 Gy) EQD2, respectively. Differences are caused by the location of the individual nodes in relation to the spatial dose distribution of IGABT, differences in total dose administered and radiobiology (HDR versus PDR).

Conclusions: The IGABT dose contribution to individual pelvic nodes depends on patient and treatment related factors, and varies considerably.

J Contemp Brachytherapy 2014; 6, 1: 21-27 DOI: 10.5114/jcb.2014.42021

Key words: brachytherapy, cervical cancer, dosimetry, HDR, MRI-guidance, PDR.

\section{Purpose}

The combination of external beam radiotherapy (EBRT), chemotherapy and brachytherapy (BT) is the standard treatment of locally advanced cervical cancer. Image-guided adaptive brachytherapy (IGABT) has been widely adopted during the last years [1,2] and guidelines have been published for treatment planning and dose reporting $[3,4]$. The main advantage is dose-volume optimization, allowing adjustments to the classical pear-shaped isodose patterns according to tumor regression during treatment and residual disease at the time of BT. Literature shows that combined EBRT and BT (especially IGABT) is curative for many patients with especially high local control rates [5-7]. However, regional recurrences do occur in the pelvic

lymph nodes (pLNN) within and adjacent to radiotherapy fields [8]. Most regional recurrences include a component of marginal failure, suggesting a deficit in target volume coverage and dose delivered to suspicious nodes [9]. From earlier publications we know that brachytherapy contributes to the pelvic nodal dose $[10,11]$, but so far there is no data reported for MRI guided IGABT.

The purpose of this study was to determine the dose contributions from image guided adaptive brachytherapy (IGABT) to individual suspicious pLNN in cervical cancer patients. Data was collected in two cancer centers, University of Pittsburgh Cancer Institute (UPCI) and University Medical Center Utrecht (UMCU).
\end{abstract}




\section{Material and methods}

We performed a combined analysis of IGABT data in two institutes; University of Pittsburgh Cancer Institute (UPCI, USA) and University Medical Center Utrecht (UMCU, The Netherlands).

\section{Patients and treatment}

\section{University of Pittsburgh Cancer Institute}

Twenty-seven patients with International Federation of Gynecology and Obstetrics (FIGO) stage IB to IIIB cervical cancer and enlarged pLNN were treated between January 2007 and December 2010 with IGABT high dose rate (HDR) applications. The patients received EBRT to the pelvis or combined pelvic and para-aortic region to 45 Gy in 25 fractions. The dose to suspicious pLNN was delivered with simultaneous integrated boosts (55 Gy in 25 fractions). Brachytherapy was scheduled once a week during the fourth or fifth week of treatment, and twice a week after completion of the EBRT. Computed tomography (CT)/magnetic resonance imaging (MRI) compatible tandem-ring applicators (Nucletron, an Elekta company, Elekta AB, Stockholm, Sweden) were used with MRI-based planning for the first BT fraction followed by CT-based planning for subsequent treatments $[6,12]$. The tandem length ranged from 40 to $60 \mathrm{~mm}$, the ringtandem angle was $45^{\circ}$ to $60^{\circ}$, and ring diameter was 26$34 \mathrm{~mm}$. All patients were treated with 5 HDR fractions of 5.0-6.0 Gy per fraction (reference dose) depending on the stage of disease and response to treatment. Planning aim was to cover $90 \%$ of the HR-CTV volume with the reference dose. Treatment planning optimization was performed using a combination of graphical and manual tools. For every patient, body mass index (BMI) was calculated.

\section{University Medical Center Utrecht}

Fifteen patients with FIGO stage IBI to IVA cervical cancer and enlarged pLNN were treated between May 2009 and September 2011. Treatment consisted of EBRT to the clinical target volume as described by van de Bunt et al. [13] combined with 3D image-guided intracavitary or combined intracavitary/interstitial pulsed dose rate (PDR) BT applications. The EBRT dose was $45 \mathrm{~Gy}$ in 25 fractions. Brachytherapy consisted of two PDR applications of 32 pulses of 0.6 Gy $(2 \times 19.2$ Gy planning aim) with the first insertion after 22 EBRT fractions, and the second one after completion of EBRT. Pulsed dose rate was delivered in a 24-hour schedule, one pulse per hour. Dose was prescribed to $90 \%$ of the HR-CTV volume. Treatment planning optimization was performed using a combination of graphical and manual tools. Brachytherapy was performed using the Utrecht Interstitial Applicator, a CT/MR compatible tandem-ovoid applicator that allows additional interstitial needle placement (Nucletron, an Elekta company, Elekta AB, Stockholm, Sweden). For the first BT fraction, only the intracavitary part of the applicator was used. In six of the fifteen patients, the dose to $90 \%$ of the HR-CTV ( $\left.\mathrm{D}_{90} \mathrm{HR}-\mathrm{CTV}\right)$ of the first application did not meet the planning aim. In this group the second application was performed with 1-6 additional interstitial needles. The number of needles and their position and depth of insertion were based on dosimetric and spatial shortcomings of the first application. After BT, all patients received an additional 3D-conformal EBRT boost of 10 to 14 Gy in 5 to 7 fractions to suspicious pLNN, taking into account the dose contribution of BT. Also for these patients, BMI was calculated.

\section{Imaging and contouring}

\section{University of Pittsburgh Cancer Institute}

At UPCI, all 27 patients had MRI examination (GE SIGNA HD excite 1.5T, GE Health Care, Milwaukee, WI, USA); T2-weighted sequences with $3.5 \mathrm{~mm}$ slice thickness for the first BT fraction. For the following four fractions, a CT examination was performed (GE LightSpeed Ultra-64 slice per rotation, GE Health Care, Milwaukee, WI, USA) with $2.5 \mathrm{~mm}$ slice thickness. CT images were acquired without intravenous contrast, but with $60 \mathrm{ml}$ diluted contrast injected into the bladder. HR-CTV and organs at risk (OAR) were contoured on an Eclipse ${ }^{\mathrm{TM}}$ workstation for each fraction, according to the GEC ESTRO recommendations $[3,4]$. Thereafter, they were transferred to the Nucletron Plato Brachytherapy Planning system (version 14.3, Nucletron, an Elekta company, Elekta AB, Stockholm, Sweden) for three-dimensional planning.

\section{University Medical Center Utrecht}

At UMCU, all fifteen patients had MRI examination for each application (Gyroscan NT Intera 1.5 T, Philips Medical Systems, Best, The Netherlands) based on the institutional protocol, which includes T2-weighted turbo spin-echo images with 3 or $4.5 \mathrm{~mm}$ slice thickness, and a balanced steady-state free precession scan with a slice thickness of $1.5 \mathrm{~mm}$ for applicator reconstruction purposes [14]. HR-CTV and OAR were contoured in VolumeTool [15] according to GEC ESTRO recommendations [3,4]. Contours were transferred into the Plato Brachytherapy Planning System, and applicator reconstruction and treatment planning was performed.

\section{Node contouring}

Gross nodal volume for each pLNN was contoured based on imaging information. Individual pLNN were contoured on the images performed for each BT fraction. Pre-treatment MRI or PET-CT information was used to guide identification of residual pLNN on BT scans. In case of complete response of the enlarged pLNN, the areas previously occupied by the individual nodes were contoured. Pelvic lymph nodes contours were generated by a specifically trained resident and verified by a radiation oncologist experienced in gynecological tumors in UPCI and UMCU, respectively.

\section{Analysis}

The dose contributions $\left(D_{90}\right)$ to each individual pLNN were calculated from dose-volume histograms (DVH) for each fraction; both as absolute physical dose 
and as percentage of the reference dose. The doses were expressed as biologically equivalent dose in 2 Gy fractions (EQD2) using LQ-model (with $\alpha / \beta=10$ for tumor, $\alpha / \beta=3$ for OAR and half time of repair of 1.5 hours) [16] and were added for all fractions. Furthermore, the $D_{90}$ for the HR-CTVs were determined in EQD2. In order to get an estimate of the influence of radiobiology, the PDR data were recalculated as if given by HDR in 4 fractions of 7 Gy and scaled for OAR constraints (clinically used HDR schedule in our institute).

For every patient, we determined the total reference air kerma (TRAK). Total reference air kerma divided by the reference dose was considered as an indicator of the volume encompassed by the $100 \%$ isodose.

\section{Anatomical nodal regions}

The contoured pLNN were grouped by three physicians into anatomical nodal regions, based on Radiation Therapy Oncology Group (RTOG) definitions of pelvic nodal groups for intensity-modulated radiation therapy (IMRT) [17] and other published literature [18-20]. The common iliac region (CI) covers the area around the common iliac vessels from the aortic to iliac bifurcation. The internal iliac region (II) is defined as an area of $7 \mathrm{~mm}$ around and along the internal iliac vessels, excluding pLNN ventrally to external iliac vessels. From the point where the internal and external iliac vessels spread to a distance of more than $7 \mathrm{~mm}$, the space around and along external iliac artery is called external iliac region (EI). The obturator region (OB) encompasses the space bordered by internal and external iliac regions.

\section{Results}

For patients characteristics see Table 1. In UPCI, 27 patients underwent 135 tandem-ring based HDR applications in total. Fifty-seven pLNN were detected with a minimum of 1 and a maximum of 9 per patient. The mean HR-CTV of these patients was $36.9 \mathrm{~cm}^{3}$ (range 21.0$59.8 \mathrm{~cm}^{3}$ ), and the mean BT D90 HR-CTV was $39.3 \mathrm{~Gy}$ (range 34.9-63.3 Gy) EQD2. The mean D90 received by the suspicious pLNN was $10.8 \%$ (range $5.7-25.1 \%$ ) of
Table 1. Patient characteristics

\begin{tabular}{lcc} 
& UPCI & UMCU \\
\hline Patients $(n)$ & 27 & 15 \\
\hline Age (years) & 49 & 53 \\
\hline mean & $29-83$ & $27-79$ \\
\hline range & & \\
\hline FIGO stage & 1 & 1 \\
\hline IB1 & 2 & 3 \\
\hline IB2 & 1 & 1 \\
\hline IIA & 17 & 4 \\
\hline IIB & 0 & 0 \\
\hline IIIA & 6 & 5 \\
\hline IIIB & 0 & 1 \\
\hline IVA & 57 & 40 \\
\hline Nodes $(n)$ & 30 & 25 \\
\hline BMI & $18-41$ & $19-32$ \\
\hline mean & & \\
\hline range & & \\
\hline
\end{tabular}

$U P C I$ - University of Pittsburgh Cancer Institute, UMCU - University Medical Center Utrecht, BMI - body mass index

the reference dose, corresponding to 2.7 Gy (range 1.36.6 Gy) EQD2. In UMCU, 15 patients underwent 30 PDR applications in total. Forty pLNN were detected with a minimum of 1 and a maximum of 5 per patient. The mean HR-CTV was $39.4 \mathrm{~cm}^{3}$ (range $15.9-115 \mathrm{~cm}^{3}$ ), and the mean BT D90 HR-CTV was 47.1 Gy (range 0.3$52.4 \mathrm{~Gy}$ ) EQD2. The mean $\mathrm{D}_{90}$ of the pLNN was $20.5 \%$ (range 6.8-93.3\%) resulting in 7.1 Gy (range 2.2-36.7 Gy) EQD2 (Table 2). Figure 1 shows an illustration of the 3D EQD2 distribution of a BT PDR fraction displaying pLNN with different locations and different dose contributions.

Table 2. Results

\begin{tabular}{|c|c|c|c|c|c|c|c|c|}
\hline & \multirow{2}{*}{\multicolumn{2}{|c|}{$\begin{array}{l}\text { UPCI } \\
\mathrm{HDR}\end{array}$}} & \multicolumn{6}{|c|}{ UMCU } \\
\hline & & & \multicolumn{4}{|c|}{ PDR } & \multirow{2}{*}{\multicolumn{2}{|c|}{$\frac{\text { Recalculated for HDR }{ }^{* *}}{\text { Selection } 35 \text { pLNN }}$}} \\
\hline & \multicolumn{2}{|c|}{57 pLNN } & \multicolumn{2}{|c|}{ All 40 pLNN } & \multicolumn{2}{|c|}{ Selection 35 pLNN } & & \\
\hline & Mean & SD & Mean & SD & Mean & SD & Mean & SD \\
\hline Volume HR-CTV $\left(\mathrm{cm}^{3}\right)$ & 36.9 & 10 & 39.4 & 28 & 39.4 & 28 & 39.4 & 28 \\
\hline $\mathrm{D}_{90}$ HR-CTV (Gy EQD2) & 39.3 & 5.2 & 47.1 & 3 & 47.1 & 3.1 & 41.2 & 4.9 \\
\hline $\mathrm{D}_{90} \mathrm{pLNN/Ref}$ dose (\%) & 10.8 & 5.1 & 20.5 & 15 & 16.7 & 7.2 & 16.7 & 7.2 \\
\hline $\mathrm{D}_{90} \mathrm{pLNN}(\mathrm{Gy}$ EQD2) & 2.7 & 1.4 & 7.1 & 5.9 & 5.6 & 2.5 & 3.9 & 1.9 \\
\hline
\end{tabular}

UPCI - University of Pittsburgh Cancer Institute, UMCU - University Medical Center Utrecht, HDR - high dose-rate, PDR - pulsed dose-rate, pLNN - pathologically enlarged pelvic lymph-nodes, SD - standard deviation, HR-CTV - high risk-CTV, $D_{90}$ - dose to $90 \%$ of the volume, ref. dose - reference dose, EQD2 - biologically equivalent dose in 2-Gy fractions

**PDR data were recalculated as if given by HDR in 4 fractions of 7 Gy and scaled for OAR constraints 

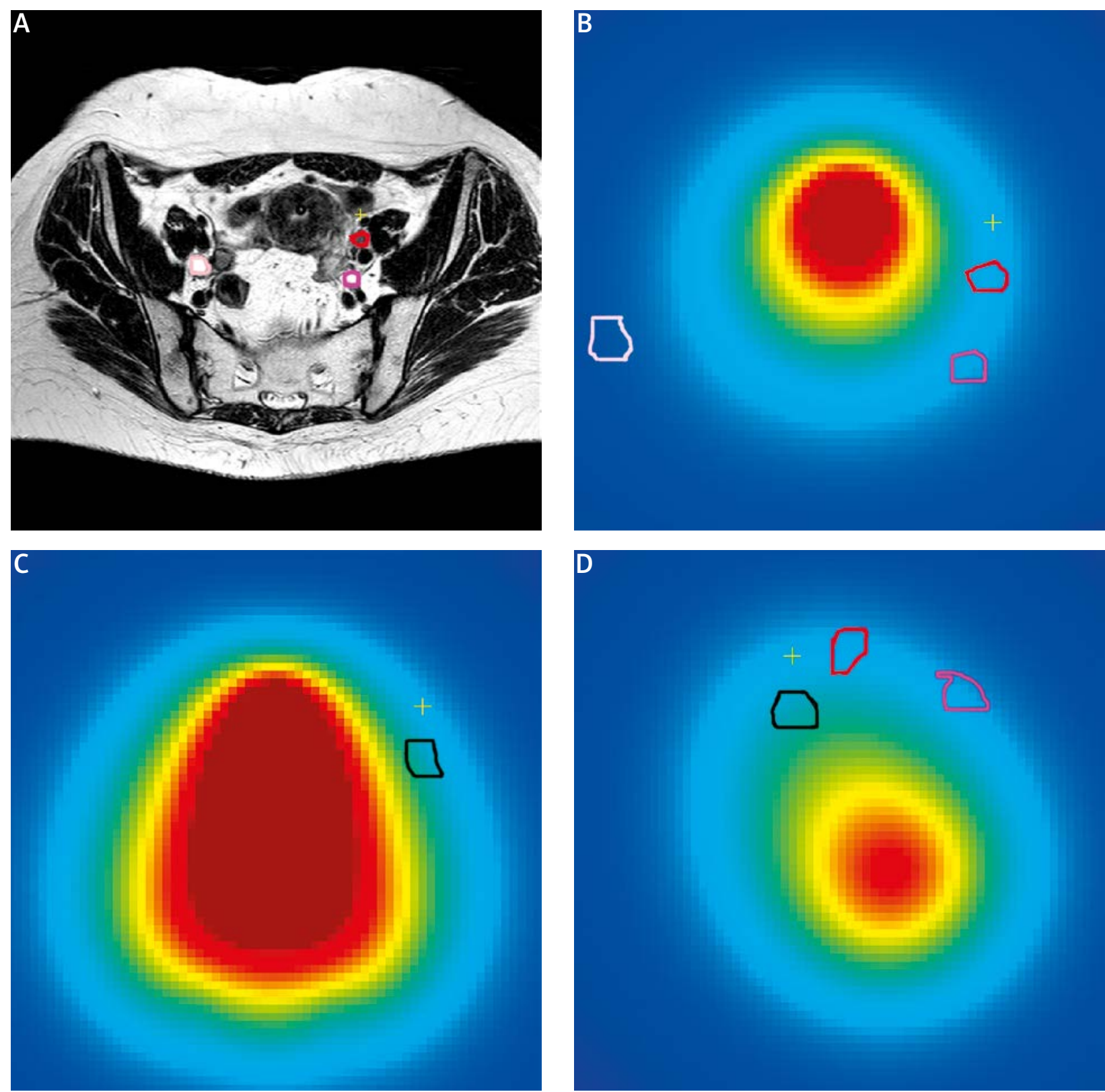

Fig. 1. Illustration of a transversal $T 2$ weighted MR image (A) and corresponding 3D dose distribution in EQD2 of a brachytherapy pulsed dose rate fraction presented in three slices: (B) transversal, (C) coronal and (D) sagittal view. Four pelvic lymph nodes are delineated (colored contours). Blue $=0$ and Red $>20$ Gy EQD2, yellow cross indicates common point for all images

Table 3 and Figure 2 show the dose contributions of IGABT to the different nodal regions. In UPCI, but not in UMCU we saw a tendency that dose decreases from obturator to external, internal and common iliac nodes. The dose contributions of IGABT to individual $\mathrm{pLNN}$ varied impressively in both centers (range $\sim 5$ to $>90 \%$ of the reference dose). After having excluded the few parametrial, inguinal and peri-rectal nodes from the UMCU data set (leaving 35 pLNN for the comparison; see Table 2), the nodes received on average $16.7 \%$ (range $6.8-34.7 \%$ ) of the reference dose, corresponding to 5.6 Gy (range 2.2-12.0 Gy) EQD2. Furthermore, recalculating the UMCU PDR into HDR values, resulted in a reduced mean D90 of 3.9 Gy (range 1.58.2 Gy) EQD2, and a smaller difference with UPCI values.
Figure 3 shows the D90 pLNN as function of TRAK per reference dose (as surrogate of the $100 \%$ isodose volume). The irradiated volumes of UMCU are in general larger than the ones of UPCI. This overall results in higher dose contributions to the pLNN in UMCU, irrespective of PDR or HDR calculations. The addition of interstitial needles for the second BT fractions in six of the UMCU patients did not influence the $\mathrm{D}_{90} \mathrm{pLNN}$ systematically; see wide range of values for those cases in Figure 3.

Body mass index was 25 (range 19-32) in UMCU patients and 30 (range 18-41) in UPCI patients, respectively, without a relation between physical $\mathrm{D}_{90}$ dose to the nodes and BMI. 
Table 3. Results for different nodal regions

\begin{tabular}{|c|c|c|c|c|c|c|c|c|}
\hline \multirow[t]{3}{*}{ Nodal region } & \multicolumn{4}{|c|}{ UPCI } & \multicolumn{4}{|c|}{ UMCU } \\
\hline & \multicolumn{4}{|c|}{$\mathrm{D}_{90} \mathrm{pLNN}(\mathrm{Gy} E Q \mathrm{E} 2)$} & \multicolumn{4}{|c|}{$\mathrm{D}_{90} \mathrm{pLNN}(\mathrm{Gy} E Q \mathrm{D} 2)$} \\
\hline & $n$ & Mean & SD & Range & $n$ & Mean & SD & Range \\
\hline Common ililac & 4 & 1.6 & 0.2 & $1.4-2.5$ & 1 & 5 & & \\
\hline Internal iliac & 14 & 2.4 & 0.8 & $1.3-6.6$ & 11 & 4.7 & 2.3 & $2.2-8.7$ \\
\hline External iliac & 32 & 2.8 & 1.4 & $1.3-6.0$ & 18 & 6.2 & 2.9 & $2.7-12.0$ \\
\hline Obturator & 7 & 3.4 & 2.2 & $1.7-5.7$ & 5 & 5.3 & 1.1 & $3.7-6.3$ \\
\hline Parametrial & 0 & & & & 3 & 22.8 & 12.2 & $14.2-36.7$ \\
\hline Inguinal & 0 & & & & 1 & 5.1 & & \\
\hline Perirectal & 0 & & & & 1 & 13 & & \\
\hline
\end{tabular}

$U P C I$ - University of Pittsburgh Cancer Institute, UMCU - University Medical University Utrecht, pLNN - pathologically enlarged pelvic lymph-nodes, $D_{90}$ - dose to $90 \%$ of the volume, EQD2 - biologically equivalent dose in 2-Gy fractions, SD - standard deviation

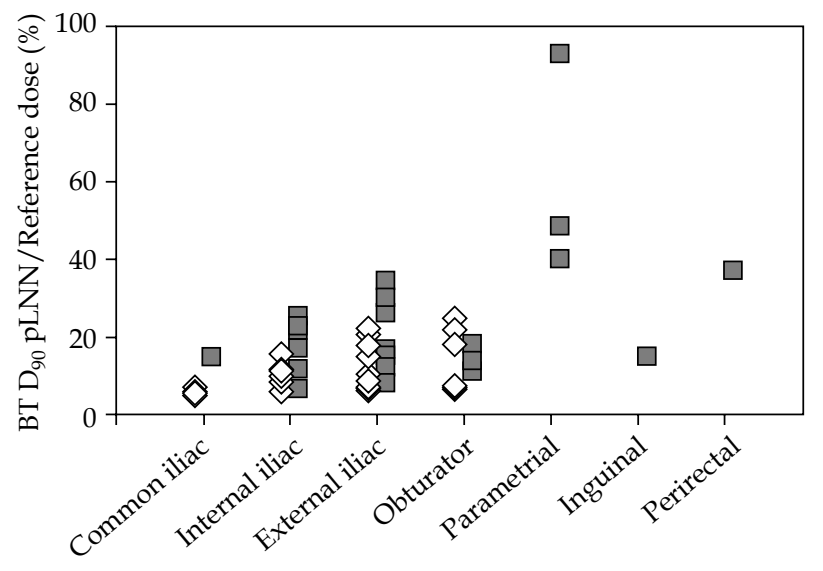

Anatomical pelvic regions

$\diamond \mathrm{UPCI} H D R \quad \square \mathrm{UMCU}$ PDR

$B T D_{90}$ pLNN - brachytherapy dose to $90 \%$ of the enlarged pelvic lymph nodes, diamonds - UPCI, grey squares - UMCU

UPCI - University of Pittsburgh Cancer Institute, UMCU - University Medical Center Utrecht

Fig. 2. Relative dose contributions of PDR and HDR treatments to individual lymph nodes in the different pelvic regions; dose variations indicated between and within anatomical regions

\section{Discussion}

To our knowledge, this retrospective inter-institutional study represents the first dosimetric analysis of MRI based IGABT dose-contributions to pelvic lymph nodes in patients with advanced cervical cancer. Since the introduction of image guided BT, local control in cervical cancer has increased [5,7], but regional control is still a matter of concern [6,9]. Additionally, modern imaging modalities allow a better visualization of pLNN [21] and help to consider them suspicious or not, which influences our intentions to treat nodal disease.

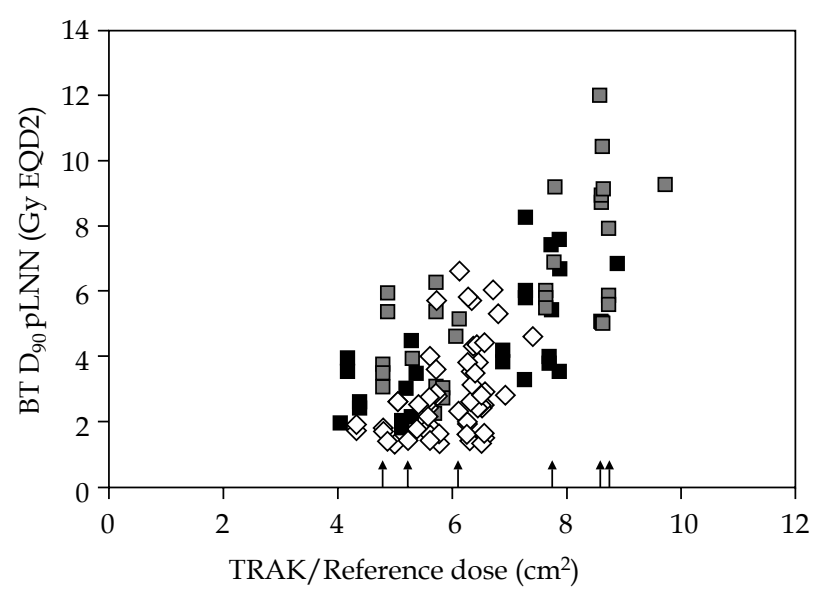

$\diamond \mathrm{UPCI} H D R \quad \square \mathrm{UMCU}$ PDR $\quad \square \mathrm{UMCU}$ HDR

$B T D_{90} p L N N$ - brachytherapy dose to $90 \%$ of the enlarged pelvic lymph nodes volumes, EQD2 - equivalent total dose in 2 Gy fractions, TRAK - total reference air kerma

Fig. 3. Dose contributions to individual lymph nodes as function of TRAK/Reference Dose; TRAK/Reference Dose is considered an indicator for the volume encompassed by the $100 \%$ isodose. Arrows indicate cases treated with a combined intracavitary/interstitial approach. Diamonds - UPCI (HDR). Grey squares - UMCU (PDR). Black squares - UMCU recalculated for HDR. The differences between black and grey squares show that the BT dose contribution (in EQD2) to pLNN is influenced by dose rate

For accurate dose reporting and for our understanding of nodal dose response relations it is essential to learn how EBRT and BT contribute to the treatment of individual affected lymph nodes. More detailed information on the dose administered per node will also help us to further optimize nodal boosting.

Isodose patterns of traditional Manchester system BT are pear shaped and symmetrical. Point B in this system was thought to give an estimate of the dose contribution 
to the lateral parametrium and the regional nodes. It is located at the level of point A, $5 \mathrm{~cm}$ lateral from the center of the tandem and gets approximately $15 \%$ to $25 \%$ of the point A dose. Lee et al. [10] already showed that the point $B$ dose is not a good surrogate for the dose to pelvic nodes. In that study, the nodal regions defined on CT scans were overlaid with the BT dose distributions showing significantly lower dose to nodal groups than to point B (on average 5 vs. 7 Gy). Additionally, it was documented that there is some variation in dose delivered to the different nodal groups, defined as internal iliac, external iliac, and obturator nodes.

In our study, we investigated the contribution of individualized IGABT to the pLNN. We computed the BT dose to individual nodes located in the different groups by using the images at time of BT and the optimized BT treatment plans. We found a variety of dose levels in individual nodes and patients, but no general dose level differences when comparing the earlier defined nodal groups. This finding might be due to the fact that the spatial dose distributions of IGABT in the central pelvis can be quite asymmetric as they follow the target and OAR locations and shapes. It can therefore be expected that individualized IGABT also individually contributes to different pLNN with the highest values to nodes in the vicinity of the HR-CTV and high dose regions. Based on the data presented here, we consider to calculate the IGABT dose for the individual nodes, especially when EBRT boosting is part of the treatment schedule. Dose calculation for the different nodal regions is a rougher estimate and might still be used if individual node dose calculation is not possible.

We found astonishing pLNN dose level differences in and between the two cancer centers. The IGABT dose contributions to pLNN in UPCI were on average lower than the ones in UMCU. Several factors were investigated in order to explain these findings. Part of the difference was caused by the fact that in some UMCU patients, different from UPCI, pLNN were identified in the vicinity of the HR-CTV, especially in parametrium and perirectally. After excluding these nodes and their corresponding high $\mathrm{D}_{90}$ values, the dose level difference was smaller. Another part of the difference is accounted for radiobiology. After having recalculated the UMCU PDR contributions to $\mathrm{pLNN}$ into HDR values, the difference in dose again was smaller, but still remained. This lasting difference is due to the fact that the prescribed and administered tumor dose in UMCU is in principal higher, irrespective of the underlying radiobiology. The on average higher $D_{90}$ HR-CTV values in UMCU are corresponding to higher totally administered doses and larger BT volumes irradiated to the reference dose.

The addition of interstitial needles to the intracavitary part of the applicator in six of the UMCU patients did not influence the dose contributions to pLNN (Fig. 3) systematically. A possible explanation is that the needles are used differently in individual situations. Some needles are intended to increase the dose to the lateral parts of the HR-CTV, others for reducing the more centrally located $\mathrm{D}_{2 \mathrm{cc}}$ values of the OAR. However, six cases with needles might not be enough to answer this question principally. Body mass index as an independent factor was investigated, but did not influence the average $D_{90}$ pLNN values. Small part of the difference might be caused by the fact that two observers contoured the nodes, one in UMCU and one in UPCI. However, after having agreed upon methodology in the first instance, both observers were instructed in one centre (UMCU). It was not possible to study a potential difference between the two applicator types as both were regularly adapted to the anatomy of the individual patients.

\section{Conclusions}

The IGABT dose contribution to individual pLNN depends on patient and treatment related factors and varies considerably. The difference is caused by the positions of the individual nodes in relation to the radiation source, by differences in radiobiology and by individual treatment planning, and spatial dose delivery to specific HR-CTV and OAR locations and shapes. Based on the data presented here, calculation of the BT dose to the individual pLNN for nodal dose reporting and individual node boost planning should be considered. Dose calculations for the different nodal regions is a rougher estimate and can be used as alternative if individual node dose calculation is not possible.

\section{Acknowledgements}

Research conducted at: Department of Radiation Oncology, University Medical Center Utrecht, The Netherlands.

\section{Disclosure}

Authors report no conflict of interest.

\section{References}

1. Dimopoulos JC, De Vos V, Berger D et al. Inter-observer comparison of target delineation for MRI-assisted cervical cancer brachytherapy: application of the GYN GEC-ESTRO recommendations. Radiother Oncol 2009; 91: 166-172.

2. Viswanathan AN, Erickson BA. Three-dimensional imaging in gynecologic brachytherapy: a survey of the American Brachytherapy Society. Int J Radiat Oncol Biol Phys 2010; 76: 104-109.

3. Haie-Meder C, Pötter R, Van Limbergen E et al. Recommendations from Gynaecological (GYN) GEC-ESTRO Working Group (I): concepts and terms in 3D image based 3D treatment planning in cervix cancer brachytherapy with emphasis on MRI assessment of GTV and CTV. Radiother Oncol 2005; 74: 235-245.

4. Pötter R, Haie-Meder C, Van Limbergen E et al. Recommendations from gynaecological (GYN) GEC ESTRO working group (II): concepts and terms in 3D image-based treatment planning in cervix cancer brachytherapy-3D dose volume parameters and aspects of 3D image-based anatomy, radiation physics, radiobiology. Radiother Oncol 2006; 78: 67-77.

5. Pötter R, Georg P, Dimopoulos JC et al. Clinical outcome of protocol based image (MRI) guided adaptive brachytherapy combined with 3D conformal radiotherapy with or without chemotherapy in patients with locally advanced cervical cancer. Radiother Oncol 2011; 100: 116-123. 
6. Beriwal S, Kannan N, Kim H et al. Three-dimensional high dose rate intracavitary image-guided brachytherapy for the treatment of cervical cancer using a hybrid magnetic resonance imaging/computed tomography approach: feasibility and early results. Clin Oncol (R Coll Radiol) 2011; 23: 685-690.

7. Nomden CN, de Leeuw AA, Roesink JM et al. Clinical outcome and dosimetric parameters of chemo-radiation including MRI guided adaptive brachytherapy with tandem-ovoid applicators for cervical cancer patients: a single institution experience. Radiother Oncol 2013; 107: 69-74.

8. Eifel PJ, Winter K, Morris M et al. Pelvic irradiation with concurrent chemotherapy versus pelvic and para-aortic irradiation for high-risk cervical cancer: an update of radiation therapy oncology group trial (RTOG) 90-01. J Clin Oncol 2004; 22: $872-880$

9. Beadle BM, Jhingran A, Yom SS et al. Patterns of regional recurrence after definitive radiotherapy for cervical cancer. Int J Radiat Oncol Biol Phys 2010; 76: 1396-1403.

10. Lee LJ, Sadow CA, Russell A et al. Correlation of point B and lymph node dose in 3D-planned high-dose-rate cervical cancer brachytherapy. Int J Radiat Oncol Biol Phys 2009; 75: 803-809.

11. Lee YC, Rash DL, Stern RL et al. The equivalent dose contribution from high-dose-rate brachytherapy to positive pelvic lymph nodes in locally advanced cervical cancer. Brachytherapy 2013; 12: 555-559.

12. Nag S, Erickson B, Thomadsen B et al. The American Brachytherapy Society recommendations for high-dose-rate brachytherapy for carcinoma of the cervix. Int J Radiat Oncol Biol Phys 2000; 48: 201-211.

13. van de Bunt $L$, van der Heide UA, Ketelaars M et al. Conventional, conformal, and intensity-modulated radiation therapy treatment planning of external beam radiotherapy for cervical cancer: The impact of tumor regression. Int I Radiat Oncol Biol Phys 2006; 64: 189-196.

14. De Leeuw AA, Moerland MA, Nomden C et al. Applicator reconstruction and applicator shifts in $3 \mathrm{D}$ MR-based PDR brachytherapy of cervical cancer. Radiother Oncol 2009; 93: 341-346.

15. Bol GH, Kotte AN, van der Heide UA et al. Simultaneous multi-modality ROI delineation in clinical practice. Comput Methods Programs Biomed 2009; 96: 133-140.

16. Lang S, Kirisits C, Dimopoulos J et al. Treatment planning for MRI assisted brachytherapy of gynecologic malignancies based on total dose constraints. Int J Radiat Oncol Biol Phys 2007; 69: 619-627.

17. Small W, Jr., Mell LK, Anderson P et al. Consensus guidelines for delineation of clinical target volume for intensity-modulated pelvic radiotherapy in postoperative treatment of endometrial and cervical cancer. Int J Radiat Oncol Biol Phys 2008; 71: 428-434.

18. Taylor A, Rockall AG, Reznek RH et al. Mapping pelvic lymph nodes: guidelines for delineation in intensity-modulated radiotherapy. Int J Radiat Oncol Biol Phys 2005; 63: 1604-1612.

19. Toita T, Ohno T, Kaneyasu Y et al. A consensus-based guideline defining the clinical target volume for pelvic lymph nodes in external beam radiotherapy for uterine cervical cancer. Jpn J Clin Oncol 2010; 40: 456-463.

20. Taylor A, Rockall AG, Powell ME. An atlas of the pelvic lymph node regions to aid radiotherapy target volume definition. Clin Oncol (R Coll Radiol) 2007; 19: 542-550.

21. Havrilesky LJ, Kulasingam SL, Matchar DB et al. FDG-PET for management of cervical and ovarian cancer. Gynecol Oncol 2005; 97: 183-191. 